\title{
Effect of Psychological Intervention on Anxiety of College Students With Pulmonary Tuberculosis
}

\author{
Hai-yan LI ${ }^{1, \text { a }}$, Feng-ju QIN ${ }^{2, \text { b }}$ \\ ${ }^{1}$ Hospital of Jinan University, Jinan City, Shandong Province, China \\ ${ }^{2}$ Hospital of Shandong University of Science and Technology, Qingdao City, Shandong Province, China \\ ${ }^{a}$ ch_lihy@ujn.edu.cn, ${ }^{b}$ qfjll66@126.com
}

\begin{abstract}
.
In this paper, firstly, to understand the psychological state of university students with tuberculosis and explore the effect of psychological intervention on the psychological anxiety of college students. Secondly, fifty-two pulmonary tuberculosis patients from a university in Jinan were randomly divided into intervention group and control group, 26 cases in each group. The psychological status of the patients was evaluated by SAS and Questionnaire of Patient's Quality of Life, and then the scores of the two groups were compared. it is concluded that the psychological anxiety of the intervention group was significantly lower than that of the control group, and the quality of life of the intervention group was significantly higher than that of the control group $(\mathrm{p}<0.01$ or 0.05$)$.Finally, understanding the psychological status of college students with tuberculosis and taking pertinent psychological intervention measures are of great positive significance to alleviate the anxiety of college students and improve their quality of life.
\end{abstract}

Keywords: Pulmonary tuberculosis, College student, Psychological intervention, Anxiety, Quality of life.

\section{Introduction}

Tuberculosis is a chronic infectious disease caused by Mycobacterium tuberculosis. In recent years, the incidence of tuberculosis is on the rise, and the prevalence of college students is also increasing. Tuberculosis has the characteristics of infectivity, long course and easy recurrence. Moreover, because of their high educational level, most of them are only children, full of confidence and fantasy about life and future, they are vulnerable to psychological anxiety and affect the quality of life. To explore the effect of psychological intervention on College Students' anxiety and quality of life after illness, self-rating anxiety scale (SAS) and patient quality of life questionnaire were used to study the anxiety level and quality of life of undergraduates with pulmonary tuberculosis after psychological intervention. The results are reported as follows.

\section{Empirical Analysis}

\subsection{Data and methods}

\subsection{1object of study}

Fifty-two pulmonary tuberculosis patients, 43 boys and 9 girls, aged 19-25 years, were selected from a university in Jinan from January 2007 to December 2008. Fifty-two college students were randomly divided into psychological intervention group and control group, 26 in each group, there was no significant difference in sex, age, physical condition, type of tuberculosis and treatment measures between the two groups. After the patient's illness is diagnosed, the school hospital should know the basic situation of the students in time and establish the file of tuberculosis patients. Because all the students have left school and been hospitalized or isolated in their homes, psychological intervention mainly adopts the way of telephone communication. Psychological intervention was carried out in the intervention group while the same medication was used in the control group. Psychological intervention personnel are professional doctors. After professional learning, they have mastered the relevant professional knowledge of tuberculosis. The following measures were taken in the psychological intervention group:(1) Care for and sympathize with patients and establish a good relationship with them.(2) Answer the patient's questions and counseling carefully and understand the patient's psychological state.(3) Give corresponding psychological support to patients' anxiety, fear, depression and disappointment.(4) Help patients understand tuberculosis correctly, introduce a series of preferential policies implemented by the state for tuberculosis patients, so that patients can be treated with ease.(5) Communicate with patients at least once a week. Understand the patient's medication, testing, psychological situation, and give patients psychological support at any time.

\subsubsection{Method of investigation}

(1) Two groups of patients followed the principle of informed and voluntary on the second day after diagnosis 
and filled in self-rating anxiety form (SAS) under the guidance of psychological intervention personnel. After one month of psychological intervention, two groups of patients were instructed to fill in SAS form again.(2) Half a year after psychological intervention, the two groups of patients were assessed with the Questionnaire of Patient's Quality of Life, a total of 12 items, with a total score of 70 points and a minimum of 35 points. The higher the score, the better the quality of life of patients.

\subsection{Result}

2.2.1There was no significant difference in anxiety between the two groups the second day in the hospital ( $p>$ 0.05). One month later, the anxiety level of psychological intervention group was significantly lower than that of control group $(p<0.01)$. The results are shown in Table 1.It shows that psychological intervention has a very positive significance in alleviating anxiety of pulmonary tuberculosis patients among college students.

Table 1. Comparison of anxiety scores between psychological intervention group and control group on the second day of admission and one month after admission $(\chi \pm s)$

\begin{tabular}{|c|c|c|}
\hline The second & day in the hospital & A month after admission to the hospital \\
\hline Psychological intervention group & $43.32 \pm 6.56$ & $31.12 \pm 5.16$ \\
\hline control group & $44.62 \pm 6.27$ & $40.45 \pm 5.56$ \\
\hline$P$ value & $>0.05$ & $<0.01$ \\
\hline \multicolumn{2}{|c|}{$\begin{array}{l}\text { 2.2.2One year later, the quality of life assessment results of } \\
\text { the two groups showed that the number of patients with } \\
\text { high score ( } 55-70 \text { points) in the intervention group was }\end{array}$} & $\begin{array}{l}\text { significantly lower than that of the control group. Both } \\
\text { have statistical significance. The results are shown in table } \\
\text { 2.It shows that psychological intervention plays a positive } \\
\text { role in improving the quality of life of patients. }\end{array}$ \\
\hline
\end{tabular}
number of people with low score $(<35$ points) was

Table 2. Quality of life assessment results (n)

\begin{tabular}{lccc} 
& & & \\
Psychological intervention group & $<35$ points & $45 \sim 55$ points & $55 \sim 70$ points \\
control group & 1 & 8 & 17 \\
P value & 3 & 13 & 10 \\
\hline & \multicolumn{3}{r}{ conquering the disease and promoting their early recovery. }
\end{tabular}

\section{Conclusion}

In recent years, there have been many outbreaks of tuberculosis outbreaks among university students in China [1] College students suffer from tuberculosis, suffer from disease, social discrimination and psychological pressure caused by economic burden, and often have different degrees of anxiety after suffering from tuberculosis. Their various psychological states have a direct impact on the prognosis of the disease. Studies have shown that [2]: Social support has a positive effect on mental health. The more social support they receive, the less anxious they will feel.

After psychological intervention in the intervention group of this study, the patients' psychological pressure was reduced, their mood was pleasant, and their anxiety was greatly improved with the cooperation of treatment and nursing. The quality of life in the intervention group was significantly higher than that in the control group. Therefore, the author believes that psychological intervention can significantly reduce the anxiety and tension of College students, alleviate the degree of psychological reaction of patients [3], and adjust the psychological state of patients. Medical workers in Colleges and universities should insist on supervising and taking medicines and regular examinations for tuberculosis patients of College students, imparting relevant knowledge of tuberculosis to them, paying attention to their psychological changes, giving psychological intervention, alleviating their anxiety, enhancing patients' confidence in

\section{References}

[1] Hongbing Ma. Disposal and analysis of an outbreak of pulmonary tuberculosis in Colleges and universities, Chinese School Physicians, 2011,25 (10): 758-759

[2] Wusen Wen, Yiqiang Wang, Guoqiu Zhao. Study on the relationship between social support, psychological control and mental health, Chinese Journal of Mental Health,2000,14:258-259

[3] Shanglun Li.Survey of prevention and treatment of tuberculosis in schools, Chinese Journal of Tuberculosis Prevention,2009,31(3):167-170 